\title{
Factores de atracción y expulsión de médicos migrantes. Estudio de caso de Pedro Aguirre Cerda
}

\author{
Attraction and expulsion factors that affect physician migration. The Pedro Aguirre Cerda case study \\ Denisse Parra-Giordano1* orcid.org/0000-0002-9439-2679 \\ Patricia Soto-Malabrigo ${ }^{1}$ orcid.org/0000-0002-0130-5995
}

1 Departamento de Enfermería. Facultad de Medicina. Universidad de Chile. Santiago-Chile

\section{Resumen}

Introducción: La migración es un fenómeno a nivel mundial que involucra a los trabajadores de las diferentes áreas como son los profesionales de la salud. Objetivo: Determinar factores de atracción-expulsión que han motivado a los médicos extranjeros a migrar escogiendo a Chile como destino, desde la perspectiva de los encargados de salud en Atención Primaria de Salud. Materiales y métodos: Estudio de caso teórico-explicativo con abordaje cualitativo en epidemiología crítica, realizado en la municipalidad de Pedro Aguirre Cerda (Chile). Se aplicó entrevistas individuales semiestructuradas a actores tomadores de decisión en la APS. Resultados: Se identificó como factores de atracción la permanencia del médico extranjero en APS, se reconoce a Chile como su hogar, relación oferta y demanda de trabajo con alta rotación. Factores expulsión: ausencia de una política de incorporación e incentivo para los médicos al servicio de APS, formación profesional diferenciada en el extranjero. Conclusiones: La identificación de factores que influyen en la atracción y expulsión de médicos en Chile, permite generar políticas públicas que mejoren las condiciones laborales de estos profesionales.

Palabras clave: Migración humana; médicos de atención primaria; atención primaria de salud; políticas públicas de salud; América Latina. (Fuente: DeCS, Bireme).

\begin{abstract}
Introduction: Migration is a worldwide phenomenon that affects workers from different areas, including health professionals. Objective: To determine the attraction-expulsion factors that have motivated foreign medical doctors to migrate to Chile, this study analyzed the perspective of those who are responsible for health care in the Primary Health Care model. Materials and methods: A theoretical-explanatory case study was carried out in the municipality of Pedro Aguirre Cerda (Chile), using a qualitative approach in critical epidemiology. Individual semi-structured interviews were administered to decision-making personnel in the Primary Health Care model. Results: The following were identified as attraction factors: (i) job stability of foreign physicians at the PHCs; (ii) recognition of Chile as their home; and (iii) the supply-demand relationship seen in highrotation jobs. On the other hand, the absence of policies of inclusion and incentives for physicians working at PHCs, and differences in medical professional training abroad were characterized as expulsion factors. Conclusions: The identification of factors that influence the attraction and expulsion of medical doctors to/from Chile contributes to generating public policies that improve the working conditions of these health professionals.
\end{abstract}

Key words: Human migration; physicians, primary care; primary health care; public health policy; Latin America. (Source: DeCS, Bireme).

\footnotetext{
*Autor de correspondencia

Denisse Parra-Giordano

e-mail: denisseparrag@gmail.com
} 


\section{Introducción}

El trabajo es uno de los aspectos fundamentales de la vida de las personas(1-3), impulsa a que los sujetos migren con la expectativa de mejorar las condiciones de vida(4-6); incluyendo en los últimos años a personal calificado como los profesionales de la salud(7,8).

La migración ha sido uno de los grandes fenómenos del siglo XXI, producto de la globalización económica y social actual(4,8-10). La teoría Push and Pull planteada por Ravenstein en los 80's, instituye 12 leyes sobre las causas de las migraciones, entendiendo que son movimientos forzados por el sistema capitalista de mercado y regulado por la oferta y la demanda(11). Push and Pull establece factores de atracción y expulsión que se basan en criterios económicos y distinto grado de desarrollo económico, inequidades que se diferencian principalmente por medio de los diferentes tipos de salarios que se puede optar, y, por lo tanto, el nivel social en el cual se vive(11). A nivel mundial la migración afecta cada vez a más países, lo cual ha traído la incorporación de este fenómeno en las agendas políticas tanto bilaterales, regionales $\mathrm{y}$ multilaterales(8,10).

Respecto a la migración calificada, desde mediados del siglo $\mathrm{XX}$, los países desarrollados han implementado diferentes medidas para dar respuesta a su demanda de personal calificado (incluidos los servicios de salud), con un aumento de la disponibilidad de profesionales que contribuyen a su crecimiento económico, en desmedro de los países pobres ${ }^{(8,10)}$. Este sistema perpetúa las diferencias entre países ricos y pobres, así, el mundo en desarrollo subsidia a los países más avanzados ocasionando sus propios déficit $(8,10)$.

En lo que respecta a Latinoamericana, los flujos migratorios han crecido en cifras, diversificando su perfil; con un $70 \%$ de su destino países dentro de la misma región $(8,10)$. Se presenta un aumento de la participación femenina, mayoritariamente jóvenes, de origen urbano y diversas clases sociales. Asimismo, se aprecia una creciente migración calificada donde predomina el personal de salud( ${ }^{(8)}$.

Cabe destacar el posicionamiento de Chile como nuevo país receptor de profesionales de salud latinoamericanos, especialmente médicos de origen ecuatoriano(8). De esta forma, Chile que era históricamente considerado país expulsor de población hasta comienzos de la década del 90, con el retorno de la democracia, se convierte en un importante destino para la migración internacional, debido al fortalecimiento de la economía, su crecimiento y estabilidad política, además de la consolidación institucional(12).

Respecto a la organización del sistema de salud en Chile, conforme a la privatización de algunos servicios básicos en los 80's, la Atención Primaria de Salud pasa a ser dependiente de las Corporaciones de Salud Municipales, las cuales, a su vez, son sujetas a cada municipalidad, donde el Director de Salud Municipal encargado corresponde a un cargo de confianza designado por el alcalde de cada municipio(13); es quien asesora al Alcalde $y$ al Concejo Municipal en la implementación de políticas relativas a salud y elaborar propuestas de mejora. Es así, como la APS constituye la puerta de entrada al sistema de salud y se centra en el Modelo de Atención Integral con Enfoque Familiar y Comunitario, que permite proporcionar a los individuos, familia y comunidad, el cuidado de la salud de manera integral, continua y centrada en el usuario(14), resolviendo casi el $80 \%$ de las atenciones en este sector, y desarrollando acciones desarrollando acciones de promoción y prevención en sus distintos niveles(15).

En algunos países los equipos de atención en salud presentan déficit de personal médico, intensificando su migración internacional(13,16). Desde 1990 en Chile el aumento de médicos extranjeros incorporados anualmente al sistema de salud obedece al déficit a nivel nacional que ha venido siendo cubierto por los médicos extranjeros(17); de manera que el $35 \%$ de médicos extranjeros laboran en 233 municipios de Chile(16).

A nivel internacional países miembros de la Organización para la Cooperación y el Desarrollo Económicos (OCDE), cuentan con ocho (Estados Unidos) o seis (Reino Unido) médicos por 10.000 habitantes, y Chile muestra 1,3 médicos por 10.000 habitantes con una recomendación nacional de tres; presentado una tasa inferior a la de médicos APS y especialistas en el sistema público de países desarrollados(18).

En Chile el déficit significativo de médicos es marcado en la APS, con una rotación constante, la cual parece tener múltiples causas como la falta de 
espacio para capacitación, anhelo del médico nacional por especializarse, limitaciones del estatuto municipal, ausencia de estabilidad laboral y salarial(19). Esta situación crítica ha llevado que casi el $50 \%$ de la dotación de médicos de la APS sean extranjeros(19), provenientes de Ecuador, Colombia, y menor concentración de Cuba, Bolivia, Perú y Venezuela.

Chile con el fin de garantizar una atención médica de calidad, desde el año 2008 estableció como requisito la presentación del Examen Único Nacional de Conocimientos de Medicina (EUNACOM) para ejercer profesionalmente la medicina en el país; la aprobación del examen habilita como médico para desempeñarse en el Sistema Nacional de Servicios de Salud, Consultorios municipales y Fuerzas Armadas y, optar a programas de especialización. En Perú donde se aplica el Examen Nacional de Medicina (ENAM), el cual es una prueba obligatoria que rinden los estudiantes de medicina al finalizar el internado médico(20). Cabe destacar que los médicos chilenos presentan una tasa de reprobación entre el 6 y 10\%, mientras que los médicos extranjeros entre $80 \mathrm{y}$ $90 \%(21)$.

A pesar de que la migración sea producida de forma voluntaria por las personas, es inducida por los factores determinados por el mercado, donde las personas migran en busca de un mejor trabajo; principalmente impulsados por ingresos mayores, lo cual se asocia a un nivel de vida superior ${ }^{(8,22)}$. La migración de personal de salud constituye un tema de preocupación creciente en América, con incipientes $\mathrm{y}$ amenazantes consecuencias para América del Sur. Sin embargo, el fenómeno de los médicos migrantes se encuentra escasamente abordado desde la perspectiva de los responsables de administrar los recursos humanos a nivel de salud pública, donde surgen interrogantes como: ¿Qué factores atraen a los médicos a migrar a Chile? ¿Qué factores expulsan a los médicos a migrar a Chile? y ¿Cómo podría mejorarse este proceso de trabajo?. Por lo anterior, el objetivo de este estudio fue determinar los factores de atracción-expulsión que han motivado a los médicos extranjeros a dejar sus países de origen, y los han llevado a escoger a Chile como destino desde la perspectiva de los encargados de salud.

\section{Materiales y métodos}

Estudio de caso teórico-explicativo desde el abordaje cualitativo con foco en la epidemiología crítica que se desarrolló en 4 etapas. Los participantes fueron cinco actores tomadores de decisión de la APS del municipio Pedro Aguirre Cerda. Conforme al Soporte Paideia estas personas que poseen un lugar de saber y poder institucional, influyen al momento de decidir sobre las políticas locales en salud, ofrecen soporte a los trabajadores de acuerdo con la realidad y contexto(23), reconociendo así las relaciones de poder existentes y sus potencialidades(24).

En la primera etapa se llevó a cabo un análisis de los tomadores de decisión del municipio donde se seleccionó al director del Departamento de Salud de Pedro Aguirre Cerda, y al director de los tres Centro de Salud Familiar (CESFAM) del municipio, CESFAM Edgardo Enríquez Fröedden, CESFAM Pierre Dubois, CESFAM Amador Negme y del Centro Comunitario de Salud Familiar (CECOSF) Miguel Enríquez.

En la segunda etapa se concertó con los participantes la fecha y lugar para la realización de la entrevista.

En la tercera etapa se llevó a cabo una caracterización de los participantes y se aplicó la entrevista, la cual contenía 12 preguntas, las cuatro primeras referentes a las demandas de los servicios y ocho preguntas relativas a los factores de atracción y expulsión de los médicos extranjeros en la APS, las cuales fueron validadas previamente por expertos en el tema. Las entrevistas tuvieron una duración entre 18 a 35 minutos, las cuales fueron grabadas y posteriormente transcritas integralmente para orientar el análisis.

En la cuarta etapa los datos fueron sometidos a análisis de contenido(25), se organizaron los datos en tres categorías desde los factores que refieren los actores entrevistados, frente a los extranjeros que trabajan con ellos, dos categorías preestablecidas: factores de atracción y factores de expulsión; y una categoría emergente: sugerencias de mejora.

Posteriormente, se conformó subcategorías y se realizó la interpretación alcanzando la saturación de la información. Finalmente, para la presentación de datos se identificó a los entrevistados con la letra E y un número según el orden en que fueron entrevistados. 


\section{Consideraciones éticas}

La confidencialidad de los datos obtenidos se informó a través de consentimientos informados, siguiendo el protocolo ético establecido por Comisión Nacional de Investigación Científica y Tecnológica de Chile (CONICYT). La entrevista no tiene costo para los entrevistados, ni se paga por la participación. Todos los participantes firmaron el consentimiento informado y se cumplieron los siete requisitos éticos Ezekiel Emanuel(26).

\section{Resultados}

Se entrevistó a cinco actores tomadores de decisión, tomadores de decisión en APS del Municipio. En relación con la caracterización sociodemográfica de la población, tres participantes son de sexo femenino y dos masculino, entre 40 y 59 años, de nacionalidad chilena quienes tienen una experiencia de trabajo en APS entre 8 y 20 años. (Tabla 1)

Tabla 1. Datos sociodemográficos actores relevantes

\begin{tabular}{ccccc}
\hline $\mathbf{N}^{\circ}$ & Sexo & Edad & Años en cargo & Años en APS \\
\hline E1 & Femenino & 53 & 6 & 20 \\
E2 & Femenino & 55 & 3 & 12 \\
E3 & Masculino & 40 & 2 & 8 \\
E4 & Masculino & 44 & 9 & 22 \\
E5 & Femenino & 59 & 3 & 20 \\
\hline & & & Media 20.6 & Media 66 \\
\hline
\end{tabular}

\section{Categoría factores de atracción}

Permanencia del médico extranjero en APS y reconoce a Chile como su hogar: Señalan que los médicos extranjeros llevan varios años desarrollando su profesión en este país, donde establecen raíces debido a que probablemente conformaron una familia y cuentan con estabilidad laboral, se desempeñan principalmente en APS debido a la mayor oferta de trabajo. Al obtener este nuevo trabajo, adaptan su nivel de vida y posición social, escribiendo su historia personal y profesional en Chile:

"En el área de la atención primaria, el impacto es enorme, ya que se viene trabajando desde hace varios años con profesionales médicos de distintos lugares de América latina, porque creo que son fenómenos que han sido paralelos y que se deben a la globalización del trabajo." E4

"Yo creo que permanecer en Chile, yo tengo un muy buen amigo extranjero, pero él ya tiene su familia consolidada en el país. Yo creo que la gran mayoría va por ahí". E2

Relación oferta y demanda de trabajo en APS con alta rotación de médicos APS: Se presenta un déficit de médicos a nivel nacional, debido a que estos se van a otros trabajos o especialidades/posgrado se presenta esta alta rotación de médicos, teniendo una alta oferta, pero baja demanda por parte de los profesionales. De esta forma, movidos por el capitalismo predominante(27), los médicos extranjeros son atraídos a Chile para tener un nivel de vida superior, y los médicos chilenos continúan su formación profesional sin retornar a la APS:

"Cómo hay falta de médicos en todo Chile los médicos que son también bastante "diostores" (se refiere al endiosamiento de las personas) se saben necesarios y empiezan a optar por plata al mejor postor el que más te ofrece." E5

"Entonces, hoy es complicado porque la oferta y la demanda están pagando mucho más en otras comunas que nosotros entonces obvio que van a preferir irse." E1

\section{Categoría factores de expulsión}

Ausencia de una política de incorporación e incentivo para los médicos al servicio de APS: Se refiere a la necesidad de una política para motivar a los médicos a ingresar y permanecer a nivel de APS, haciéndose imperioso implementar políticas públicas con el propósito de retener a estos profesionales(28). De este modo, quebrar el círculo capitalista actual, motivándolos a trabajar en APS:

"Mira nosotros llevamos meses viendo el tema a nivel comunal, con el consejo de salud ver como incentivamos a los médicos que se queden... ahora tengo una doctora que renuncia el primero de Julio y se va a otro lado porque le pagan más." E5

“Creo que donde más hemos tenido problema es que tenemos diferencias dadas por las diferentes políticas que tienen los extranjeros de ejercer en nuestro país, por un lado, tenemos tratados que permiten que algunos extranjeros de algunos países ingresen $e$ inmediatamente ingresen al mercado laboral y hay otros países a los que a los que se les pone muchas barreras." E4 
Formación profesional médica diferenciada en el extranjero: La formación profesional que existe en medicina en las universidades extranjeras es diferente, tanto a nivel nacional en cada país como al comparar entre ellos. Esto debido a los distintos grados de desarrollo de los países, y sus niveles de calidad en educación, dejándolos como países emisores o receptores de migrantes médicos conforme al mercado capitalista actual en Latinoamérica. Así, en Chile los programas de medicina son de duración de 6 a 7 años, pasando por programas de acreditación además de un examen nacional de habilitación(29).

"Los que más recibimos son ecuatorianos, entre ellos existe diferencias. Hay unos que vienen de Quito y otros de Guayaquil, se da cuenta que las exigencias son distintas." E4

"La formación médica ecuatoriana no me gusta, o sea no es que no me guste, encuentro que hay falencias, $y$ bueno muchos de ellos no tienen mucha formación en lo respiratorio y tienen que adaptarse. Los cubanos se adecuan y se ven bien no hay drama; los chilenos se ven bien, digamos que ellos tienen su prestancia, y los colombianos, si también tenemos médicos colombianos bastante buenos." E3

\section{Categoría sugerencias para mejorar la participación de médicos extranjeros en APS}

"Nivelación a través del EUNACOM a profesionales médicos". Al igual que otros países, tener una exigencia que evalúe tanto las capacidades profesionales de los médicos nacionales como extranjeros, reconociendo la nivelación como la posibilidad de dar reconocimiento frente a las evaluaciones a nivel público y privado.

"Entonces creo que se debe establecer un mecanismo que sea para todos igual y que evaluara su desempeño y que haga un reconocimiento de su evaluación." E4

"Que se les exigieran los mismos niveles de formación, que los médicos nacionales que entraran al examen de médicos nacional y que se mejoraran las políticas en cuanto a la calidad de los médicos y en cuanto a la verificación de especialización que tengan los médicos también". E2

“Trabajo coordinado Municipio y Ministerio de Salud para generación de políticas en APS". A nivel nacional se deben desarrollar planes y generación de políticas respecto al trabajo de médicos a nivel de APS, así, el Estado debe tomar parte activa en coordinación con los municipios, ejerciendo su rol de soberanía, de manera que la población acceda a una salud de calidad.

"Si de hecho siempre deberían generarse políticas nuevas con nuevos incentivos tanto para los nacionales como para los extranjeros para venir a trabajar en la Atención Primaria de Salud. Nunca esta demás." E2

"A los médicos extranjeros les haría una pasantía desde el ministerio, los mandaría como becados a diferentes lugares a donde ellos se van a capacitar y luego los evaluaría, pero en terreno, y luego que los manden a los municipios, coordinándolo todo." E3

\section{Discusión}

El ejercicio de la profesión médica en el mundo globalizado actual se enfrenta a la constante motivación por alcanzar un nivel de vida superior, que se materializa con la realización de un postgrado, con el cambio de trabajo a nivel nacional, o como migración al extranjero cuando el país de origen no ofrece posibilidades. En una investigación con estudiantes de medicina de nueve países latinoamericanos, casi la mitad desea ejercer su profesión fuera del país donde estudió(24), en España incluso más de la mitad refiere este $\operatorname{deseo}^{(9)}$ y el $15,4 \%$ no regresaría al país posteriormente(24); evidenciando que la decisión de migrar del profesional médico surge de aspiraciones como estudiante, incluso de motivaciones para estudiar la carrera como medio para poder migrar.

El factor más importante que motiva a los sujetos a migrar es la dificultad de alcanzar el nivel de vida y bienestar deseado en su país de origen, dado que el empleo y salud son aspectos determinantes en el bienestar del individuo(10,30). Según un informe de la OCDE, los profesionales son motivados a migrar en busca de mejor remuneración o mayores beneficios económicos ofertados en el nuevo escenario(6,10,31-33). Además, es importante considerar que la migración de médicos no solamente proviene desde países pobres, sino también de países hacia un país con mayor demanda y mejores condiciones de trabajo(6,9).

Es así como los médicos presentan un alto motivo de migrar por razones laborales, principalmente para conciliar la vida personal y laboral, tener mayor 
remuneración, la posibilidad de educación continua, participar de un mejor sistema de salud y acceder a más recursos para su práctica profesional(4). Finalmente, se plantea la creación de becas de retorno, de tal forma, los migrantes puedan volver a su país(5), pero reconociendo el derecho de cada profesional a decidir su forma de desarrollarse personal y profesionalmente ${ }^{(6)}$. La Organización Panamericana de la Salud (OPS), plantea la urgente necesidad de crear y ejecutar estrategias de retorno para los profesionales que migraron(8), reconociendo que aportan al desarrollo del país de origen así como al receptor(10).

En un estudio con relación al ejercicio de la medicina en la APS sobre dos tercios de estudiantes de medicina de nueve países latinoamericanos refieren que desean realizar labores de atención hospitalaria, y menos de la mitad manifiesta deseos de trabajar en APS(24), a nivel nacional se plantea un desinterés de los médicos de trabajar en APS(21). Sin embargo, en el presente estudio, trabajar en APS constituye un factor de atracción de médicos extranjeros. Por lo anterior, se observa que muchos médicos permanecen durante periodos cortos en APS, presentándose una alta rotación que no permite a los médicos conocer a la población y aplicar la capacitación recibida ${ }^{(19) .}$

En Chile existe la creencia de que una de las principales razones por las cuales los médicos se desempeñan en APS, se debe a que se encuentran en periodo de transición previa a cursar una especialidad(24). Asimismo, en Venezuela la especialización también se plantea como un motivo para la migración de médicos ${ }^{(4)}$. Por otro lado, estudiantes de medicina también mencionan su percepción de que los médicos en APS tienen una menor preparación o no cuentan con otra opción laboral y que esta actividad ofrece menor prestigio en la sociedad y en el gremio médico(24); un estudio realizado en Venezuela plantea la falta de reconocimiento social como una motivación para migrar fuera del país(4), dado que en el contexto internacional la medicina se posiciona como una de las profesiones con mayor reconocimiento $\mathrm{y}$ remuneración.

Actualmente, se presenta una gran oferta laboral para médicos en APS, sin embargo, esto será revertido en los próximos años, debido al número de egresados de las escuelas de medicina en diversos países, por ejemplo, en España se ha calculado que el déficit será corregido en los próximos 20 años(9). En lo relativo a la oferta y demanda de médicos en APS, cabe destacar que el Servicio de Salud Sur, al cual pertenece al municipio en estudio, presentó una de las mayores dotaciones de médicos a nivel nacional(19), resaltando la importancia de planificar la oferta de profesionales y así responder a las necesidades de la población(34).

Existe una suma de factores indispensables para conseguir la adherencia de los médicos en la APS, como calidad de los recintos, disponibilidad de recursos tecnológicos, expectativas salariales, posibilidad de desarrollo profesional y educación continua(21). Una investigación con estudiantes de medicina, identifica a la APS como rutinaria, limitada, con problemas de infraestructura y casos poco interesantes(24). Sin embargo, estos elementos son considerados como menores en el panorama general de los migrantes, quienes se plantean un objetivo que trasciende las condiciones laborales.

Igualmente, es importante reconocer que en Chile debido al modelo económico neoliberal vigente se privatizaron diferentes servicios de necesidad básica como la salud(35), de tal forma los trabajadores desarrollan su rol sin protestar frente a las condiciones(36). En Chile el marco jurídico de funcionamiento de la APS se encuentra definido en estatutos gubernamentales que rigen el funcionamiento de las corporaciones municipales de salud(13). Así, se establece la remuneración a los médicos, a partir de un pago fijo y unos adicionales por otras responsabilidades(13). Lo anterior demanda evaluar los ingresos económicos conforme a la variable densidad de médicos disponibles en cada zona y elaborar planes adecuados para cada zona(37), equilibrando el desbalance existente entre las zonas públicas-privadas y zonas rurales(38).

Por otro lado, un estudio realizado en Perú refiere que los médicos registran ingresos menores a los reportados en otros países(37), reconociendo que el salario es un factor influyente en la satisfacción del profesional y en la decisión de migrar a otro país; sumado a esto la mayoría de estudiantes de medicina de nueve países de Latinoamérica refieren que los médicos de APS tienen menores ingresos que quienes trabajan en hospital, incluso más de la mitad manifiesta que si trabajara en APS la remuneración no permitiría satisfacer sus necesidades económicas(24). De esta forma, los bajos salarios se presentan como una motivación para migrar fuera 
del país, asimismo, la falta de incentivos para permanecer $(4,6,8)$.

Con relación a los factores de expulsión al igual que en esta investigación se plantea la necesidad de una política de incorporación e incentivo para los médicos al servicio de $\operatorname{APS}(7,16)$. Bajo la primicia del déficit actual de personal de salud capacitado(39), la carencia actual de médicos agravada por ausencia de políticas públicas que promuevan su retención, la llegada de médicos extranjeros se incrementa ${ }^{(8,19)}$. En Venezuela se plantean como estímulos para que los médicos migren fuera del país, la ausencia de estrategias para reclutar y mantener a los profesionales ejerciendo en su territorio(4).

En cuanto a la realidad mundial, un estudio en India refiere que la escasez de trabajadores de salud calificados en un país puede deberse a la migración y a la ausencia de políticas de capacitación, contratación y retención( ${ }^{(33)}$. La OPS refiere para la Subregión Andina que las políticas locales deben ser capaces de ofertar un programa de incentivo capaz de competir con los otros países, considerando en primera instancia la fijación del costo de retención en cada país(8).

Continuando con la contribución a la expulsión desde los países de origen, se evidenció una Formación profesional diferenciada en el extranjero. Existe una diferencia en la formación de los médicos a nivel latinoamericano(8,40), en las últimas dos décadas los sistemas educativos han desarrollado un proceso de diversificación tanto en organización, modelos universitarios, y más aún, en su calidad(40). Para la Subregión Andina la OPS plantea que se debe realizar un proceso de integración subregional frente al reconocimiento de equivalencia académica de títulos y su convalidación para el ejercicio profesional; con un gran potencial para lograr una articulación educativa(8). Desafíos/dificultades enfrentadas en la gestión en la Atención Primaria a la Salud, los hallazgos señalan que el modelo de atención en la Atención Primaria a la Salud es complejo y desafiador, tanto para formuladores de políticas como para quien realiza la gestión(41).

Se reconoce el rol histórico que han tenido los médicos extranjeros para elevar las condiciones de salud nacional(21), siendo importante el nivel de preparación médica en APS(40), en el pregrado y el posgrado(42).
A nivel internacional la OCDE plantea la necesidad de establecer un examen de nivelación para los médicos, con énfasis en el idioma local, conocimiento de enfermedades prevalentes y una práctica supervisada para ejercer la profesión o especialidad determinada(21). En Chile, EUNACOM sería la institución encargada de ofrecer una nivelación previa al examen de validación y reducir así, la tasa de reprobación de médicos extranjeros ${ }^{(43)}$. Así, el migrante seducido por el mercado, se encuentra en igualdad de condiciones.

Dicha nivelación de profesionales, sumada al trabajo coordinado entre los municipios y el Ministerio de Salud, garantizaría la efectividad del sistema de salud basado en APS, la prestación de servicios de calidad con costos razonables(15), que da respuesta al consenso latinoamericano(34). La OCDE ha sugerido a los países receptores de médicos migrantes ampliar su capacidad de formación y mejorar políticas que favorezcan la mejora de la fuerza laboral nacional, a la vez, así como las condiciones de trabajo y gestión de recursos humanos(38). Frente a esto, un estudio venezolano recomienda fortalecer la gestión y el desarrollo profesional de los médicos, para concertar las expectativas con las necesidades de salud dentro de un marco jurídico(4). Asimismo, un estudio en Jamaica manifiesta la importancia del rol el Ministerio de Salud en el diálogo con los trabajadores de salud para el éxito de los programas $^{(44) \text {. }}$

A nivel mundial existe un consenso sobre la necesidad de renovar la APS en las Américas, adaptándose a los contextos sociales, culturales y económicos de cada país(42), lo cual exige redefinir el rol del médico dentro del Sistema Público de Atención, así instaurar una relación entre la formación en medicina y las normas de dotación de los servicios(19), de manera que los encargados de la gestión municipal brinden soporte a todo el recursos humano ejerciendo con responsabilidad su rol en el proceso de trabajo(23).

\section{Conclusiones}

El desarrollo del presente estudio permitió determinar desde la perspectiva de los encargados a nivel municipal de la APS, como factores de atracción: la permanencia del médico extranjero en APS y reconocimiento de Chile como su hogar, relación oferta y demanda de trabajo en APS con alta rotación de médicos APS; y como factores de 
expulsión: ausencia de una política de incorporación e incentivo para los médicos, formación profesional diferenciada en el extranjero; y también sugerencias de mejora: nivelación a través del EUNACOM a profesionales médicos, trabajo coordinado Municipio y Ministerio de Salud para generación de políticas en APS. Estos elementos han motivado a los médicos extranjeros a dejar su país de origen, y los han llevado a escoger a Chile como destino, logrando el objetivo general planteado.

Desde la epidemiología, la identificación de factores que influyen en la atracción y expulsión de médicos en Chile permite generar políticas públicas que mejoren las condiciones laborales de estos profesionales.

\section{Referencias}

1. Parra Giordano D, Felli VEA. Work process of nursing professors. Rev Lat Am Enfermagem [Internet]. 2017 [citado 20 de marzo de 2020];25. Disponible en: http://www.scielo.br/scielo.php?script=sci_abstract\&pid=S 0104-11692017000100392\&lng=en\&nrm=iso\&tlng=en

2. Gobierno de Chile M de S (MINSAL). Primera Encuesta Nacional de Empleo, Trabajo, Salud y Calidad de Vida de los Trabajadores y Trabajadoras en Chile (ENETS 2009-2010) [Internet]. Disponible en: https://www.isl.gob.cl/wpcontent/uploads/2011/09/Informe-Final-ENETS-

Interinstitucional.pdf

3. Silva COD, Ramminger T. O trabalho como operador de saúde. Ciênc Saúde Coletiva [Internet]. 2014 [citado 20 de marzo de 2020];19:4751-4758. Disponible en: https://www.scielosp.org/scielo.php?pid=S141381232014001204751\&script=sci_arttext

4. Hernández T, Ortiz Gómez Y. La migración de médicos en Venezuela. 2011 [citado 20 de marzo de 2020]; Disponible en: http://iris.paho.org/xmlui/handle/123456789/9466

5. Leal D. Bienestar, capital social y migración internacional. El caso de la España latinoamericana y andina. Migr Publ Inst Univ Estud Sobre Migr [Internet]. 2014 [citado 20 de marzo de 2020];(36):369-401. Disponible en: https://revistas.comillas.edu/index.php/revistamigracione s/article/download/4800/462

6. Parsi K. International medical graduates and global migration of physicians: fairness, equity, and justice. Medscape J Med [Internet]. 2008 [citado 20 de marzo de 2020];10(12):284. Disponible en: https://www.ncbi.nlm.nih.gov/pmc/articles/pmc2643999/

7. Stanczyk L. Managing skilled migration. Ethics Glob Polit [Internet]. 2016 [citado 20 de marzo de 2020];9(1):33502. Disponible en: https://www.tandfonline.com/doi/full/10.3402/egp.v9.33 502

8. Organización Panamericana de Salud. Migración Calificada en Salud, Impacto Financiero, Reconocimiento de Títulos: Retos y Perspectivas en los Países de la Región Andina [Internet]. 2013 [citado 20 de marzo de 2020]. Disponible en: https://www.paho.org/hq/dmdocuments/2013/RHmigracali-subreg-andina-2013.pdf
9. Bernardini-Zambrini D, Barengo N, Bardach A, Hanna M, Núnez JM. ¿Migrar o no migrar? ¿Qué pasará con nuestra próxima generación de médicos? Estudio sobre causas y motivos en estudiantes avanzados de medicina en 11 universidades de España. Aten Primaria [Internet]. 2011 [citado 20 de marzo de 2020];43(5):222-226. Disponible en:

http://www.sciencedirect.com/science/article/pii/S02126 56710001071

10. Lotero-Echeverri G, Pérez Rodríguez MA. Migraciones en la sociedad contemporánea: Correlación entre migración y desarrollo. RETOS Rev Cienc Adm Econ [Internet]. 2019 [citado 20 de marzo de 2020];9(17):145-159. Disponible en:

http://scielo.senescyt.gob.ec/scielo.php?script=sci_arttext\& pid=S1390-86182019000100145

11. Ravenstein EG. The laws of migration. J Stat Soc Lond [Internet]. 1885 [citado 20 de marzo de 2020];48(2):167235. Disponible en: http://www.jstor.org/stable/2979181

12. Gobierno de Chile. Departamento de Extranjería y Migración del Ministerio, del Interior y Seguridad Pública. Migración en Chile 2005 - 2014 [Internet]. Migraciones Chile; 2016 [citado 20 de marzo de 2020]. Disponible en: www.extranjeria.gob.cl/media/2016/06/Anuario.pdf

13. Gobierno de Chile. Ministerio de Salud. Marco Jurídico de la Atención Primaria de Salud Municipal: compilación de leyes y reglamentos de Atención Primaria de Salud Municipal No37 [Internet]. 2011 [citado 20 de marzo de 2020]. Disponible en: http://www.bibliotecaminsal.cl/wp/wpcontent/uploads/2016/03/37.pdf

14. Parra-Giordano D. Revolución científica de la salud familiar en Chile. Rev Enferm Actual En Costa Rica [Internet]. 2016 [citado 20 de marzo de 2020];31(1-16). Disponible en: https://www.scielo.sa.cr/pdf/enfermeria/n31/1409-4568enfermeria-31-00243.pdf

15. Osorio AG, Álvarez CV. La Atención Primaria de Salud: desafíos para su implementación en América Latina. Aten Primaria [Internet]. 2013 [citado 20 de marzo de 2020];45(7):384-392. Disponible en: https://www.sciencedirect.com/science/article/pii/S0212 656713000413

16. Silva N, Rebolledo C, Arteaga O, Bass C. Propuesta para identificar la brecha de médicos en la dotación de atención primaria de Salud Municipal. Esc Salud Pública Fac Med Univ Chile. 2014.

17. Rojas N \& Silva C. Observatorio Iberoamericano sobre Movilidad Humana, Migraciones y Desarrollo, OBIMID [Internet]. Instituto Universitario de Estudios sobre Migraciones. Universidad Pontificia Comillas. Madrid, España.; 2016 [citado 20 de marzo de 2020]. Disponible en: http://www.extranjeria.gob.cl/media/2016/08/informe_ju lio_agosto_2016.pdf

18. Rojas MP, ScharagerJ EA, Puschel K, Mora I, Hidalgo G. Estimación de brechas de especialistas en medicina familiar para la atención primaria chilena. Concurso Políticas Públicas-Propues Para Chile Santiago Chile Pontif Univ Católica Chile [Internet]. 2011 [citado 20 de marzo de 2020]; Disponible en: https://politicaspublicas.uc.cl/wpcontent/uploads/2015/02/estimacion-de-brechas-deespecialistas-en-medicina-familiar-para-la-atencionprimaria-chilena.pdf

19. Montoya-Aguilar C. Médicos en el nivel primario del sistema público de atención de salud de Chile: tendencia, distribución geográfica y normas. Cuad Med Soc Chile 
[Internet]. 2008 [citado 20 de marzo de 2020];48(4):215$25 . \quad$ Disponible en: http://cms.colegiomedico.cl/Magazine/2008/48/4/48_4_3. pdf

20. Mejia CR, Ruiz-Urbina FN, Benites-Gamboa D, AlbitresFlores L, Mena LS, Fasanando-Vela R. Percepciones de utilidad y preparación para el Examen Nacional de Medicina en 10 facultades peruanas, 2017. Educ Médica [Internet]. 2019 [citado 20 de marzo de 2020];20:124-130. Disponible en:

https://www.sciencedirect.com/science/article/pii/S1575 181318300184

21. Reyes H, Norero C, Pinto ME, Llanos O, Morán S, O’Ryan M, et al. Control de calidad de los médicos que inician el ejercicio profesional en Chile: Declaración de la Academia Chilena de Medicina del Instituto de Chile. Rev Médica Chile [Internet]. 2016 [citado 20 de marzo de 2020];144(5):656$657 . \quad$ Disponible en: http://www.scielo.cl/scielo.php?pid=S003498872016000500014\&script=sci_arttext

22. Unión Interparlamentaria, la Organización Internacional del Trabajo y las Naciones Unidas (Oficina del Alto Comisionado para los Derechos Humanos). Migración, derechos humanos y gobernanza. Manual para Parlamentarios $\mathrm{N}^{\circ} 24$. [Internet]. 2015 [citado 20 de marzo de 2020]. Disponible en:

https://www.ohchr.org/Documents/Publications/Migratio nHR_and_Governance_HR_PUB_15_3_SP.pdf

23. Campos GWS. Paideia y gestión: un ensayo sobre el soporte Paideia en el trabajo en salud. Salud Colect [Internet]. 2005 [citado 20 de marzo de 2020];1:59-67. Disponible en: https://www.scielosp.org/scielo.php?pid=S1851$82652005000100004 \&$ script=sci_arttext\&tlng=en

24. Mayta-Tristán P, Carbajal-Gonzalez D, Mezones-Holguín E, Mejia CR, Pereyra-Elías R, Villafuerte-Gálvez J, et al. Situación actual y perspectivas profesionales de los estudiantes de medicina de nueve países de Latinoamérica, 2008: estudio preliminar. CIMEL Cienc E Investig Médica Estud Latinoam [Internet]. 2010 [citado 20 de marzo de 2020];15(1). Disponible en: https://www.cimel.felsocem.net/index.php/CIMEL/article/ download/65/70

25. Bardin L. Análise de Conteúdo. São Paulo: Edições 70; 2011.

26. Emanuel EJ, Wendler D, Killen J, Grady C. What makes clinical research in developing countries ethical? The benchmarks of ethical research. J Infect Dis [Internet]. 2004 [citado 16 de marzo de 2020];189(5):930-937. Disponible en: https://academic.oup.com/jid/articlepdf/189/5/930/6024850/189-5-930.pdf

27. Canales AI, Pizzonia CB. Movilidad, migraciones y trabajo en el capitalismo global - Anthropos. Cuadernos de cultura crítica y conocimiento. [Internet]. Nariño SL: Anthropos Editorial; 2019 [citado 20 de marzo de 2020]. 208 p. Disponible en: https://issuu.com/anthroposeditorial/docs/ra_251_issuu

28. Colegio Médico de Chile. Propuestas para potenciar la Salud Pública [Internet]. 2014 [citado 20 de marzo de 2020]. Disponible en: http://revista.colegiomedico.cl/propuestaspara-potenciar-la-salud-publica/

29. Noriega LM, Castillo M, Santolaya ME. Formación de especialistas en Latinoamérica. Rol de las universidades chilenas. Educ Médica [Internet]. 2018 [citado 20 de marzo de 2020];19:25-30. Disponible en:
https://www.sciencedirect.com/science/article/pii/S1575 181318300275

30. Durand M. The OECD Better Life Initiative: How's Life? and the Measurement of Well-Being. Rev Income Wealth [Internet]. 2015 [citado 20 de marzo de 2020];61(1):4-17. Disponible en: https://onlinelibrary.wiley.com/doi/abs/10.1111/roiw.12 156

31. Hernandez LJ. Que critica la epidemiología crítica: una aproximación a la mirada de Naomar Almeida Filho. Bol Obs En Salud [Internet]. 2009 [citado 20 de marzo de 2020];2(4). Disponible en: http://www.revistas.unal.edu.co/index.php/bos/article/do wnload/10453/10943

32. Gil-Alonso F, Vidal-Coso E. Inmigrantes Extranjeros en el mercado de trabajo español: ¿Más resilientes o más vulnerables al impacto de la crisis? Migr Publ Inst Univ Estud Sobre Migr [Internet]. 2015;(37):97-123. Disponible en:

https://www.razonyfe.org/index.php/revistamigraciones/a rticle/viewFile/5510/5319

33. Walton-Roberts M, Runnels V, Rajan SI, Sood A, Nair S, Thomas $\mathrm{P}$, et al. Causes, consequences, and policy responses to the migration of health workers: key findings from India. Hum Resour Health [Internet]. 2017 [citado 20 de marzo de 2020];15(1):28. Disponible en: https://human-resourceshealth.biomedcentral.com/articles/10.1186/s12960-0170199-y

34. Haggerty JL, Yavich N, Báscolo EP. Un marco de evaluación de la atención primaria de salud en América Latina. 2009 [citado 20 de marzo de 2020]; Disponible en: http://iris.paho.org/xmlui/handle/123456789/9754

35. Saavedra E. El modelo económico-político de Chile: Desarrollo institucional en la encrucijada. Econ Política [Internet]. 2014 [citado 20 de marzo de 2020];1(1):115$146 . \quad$ Disponible en: https://dialnet.unirioja.es/descarga/articulo/6266406.pdf

36. Breilh J. Salud pública, universidad y poder. La universidad que queremos. Rev Fac Nac Salud Pública [Internet]. 2019 [citado 20 de marzo de 2020];1(37):7-13. Disponible en: http://repositorio.uasb.edu.ec/bitstream/10644/6478/1/B reilh\%2C\%20J.-CON-263-Salud\%20publica.pdf

37. Taype-Rondan A, Torres-Roman JS, Herrera-Añazco P, Diaz CA, Brañez-Condorena A, Moscoso-Porras MG. Ingresos económicos en médicos peruanos según especialidad: un análisis transversal de la ENSUSALUD 2015. Rev Peru Med Exp Salud Publica [Internet]. 2017 [citado 20 de marzo de 2020];34(2):183-191. Disponible en: http://www.scielo.org.pe/scielo.php?pid=S172646342017000200004\&script=sci_arttext\&tlng=en

38. Co-operation O for E, Development. International Migration of Health Workers: Improving international co-operation to address the global health workforce crisis [Internet]. OECD Paris; 2010 [citado 20 de marzo de 2020]. Disponible en: https://www.who.int/hrh/resources/oecdwho_policy_brief_en.pdf

39. Labonté R, Sanders D, Mathole T, Crush J, Chikanda A, Dambisya $Y$, et al. Health worker migration from South Africa: causes, consequences and policy responses. Hum Resour Health [Internet]. 2015 [citado 20 de marzo de 2020];13(1):92. Disponible en: https://human-resourceshealth.biomedcentral.com/articles/10.1186/s12960-0150093-4 
40. Pinzón Flórez CE. Los grandes paradigmas de la educación médica en Latinoamérica. Acta Médica Colomb [Internet]. 2008 [citado 20 de marzo de 2020];33(1). Disponible en: https://www.redalyc.org/pdf/1631/163113349007.pdf

41. Pires DEP de, Vandresen L, Machado F, Machado RR, Amadigi FR. Primary healthcare management: What is discussed in the literature. Texto Contexto-Enferm [Internet]. 2019 [citado 20 de marzo de 2020];28. Disponible en: http://www.scielo.br/scielo.php?pid=S010407072019000100501\&script=sci_arttext

42. Rojas Ochoa F. La muy necesaria renovación de la Atención Primaria de Salud. Rev Cuba Salud Pública [Internet]. 2009 [citado 20 de marzo de 2020];35(4). Disponible en: http://scielo.sld.cu/scielo.php?script=sci_arttext\&pid=S086 4-34662009000400001

43. Departamento de estudios, extensión y publicaciones. Asesoría técnica parlamentaria: El EUNACOM y la habilitación de médicos titulados en el exterior [Internet]. Biblioteca del Congreso Nacional de Chile; 2017 [citado 20 de marzo de 2020]. Disponible en: https://www.bcn.cl/obtienearchivo?id=repositorio/10221/ 24727/1/BCN_EG_Eunacom_y_medicos_extranj_20170404. pdf

44. Murphy GT, MacKenzie A, Waysome B, Guy-Walker J, Palmer $\mathrm{R}$, Rose $\mathrm{AE}$, et al. A mixed-methods study of health worker migration from Jamaica. Hum Resour Health [Internet]. 2016 [citado 20 de marzo de 2020];14(1):36. Disponible en: https://human-resources-

health.biomedcentral.com/articles/10.1186/s12960-016-

0125-8 